\title{
Semiconductive Properties of Stannous Acetate
}

\author{
Masaru OKUYAMA*, Eiichi KAWAMATA* and Satoshi KANBE*
}

\section{Introduction}

Some of the authors ${ }^{1 \sim 3}$ developed a new method for production of semiconductive transparent thin films of stannic oxide through the thermal decomposition of stannous acetate, Sn $\left(\mathrm{CH}_{3} \mathrm{COO}\right)_{3}$. In the course of developement, the authors found out that stannous acetate indicated the semiconductive properties. In the present work, the electric conductivity and the thermoelectric motive force were measured on the pressed stannous acetate powder samples. The doping effect of various solvents in them was also examined. It was found that stannous acetate exhibited excellent sensitivity to the temperature change and that its electric conductivity increased by doping polar solvents in

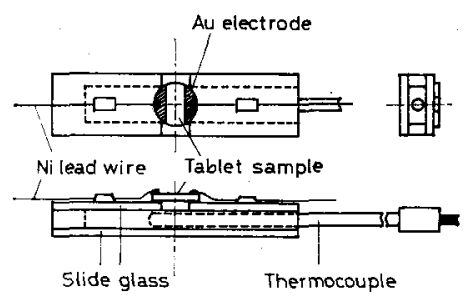

(A)

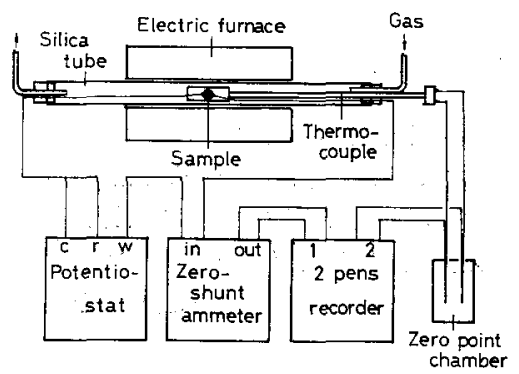

( B)

Fig. 1 Schematic diagram of sample and sample holder (A) and electric circuit for electric conductivity measurement

\footnotetext{
* Oyama Technical College (771 Nakaguki, Oyama-shi, Tochigi)

Key Words: Semiconductor, Stannous Acetate
}

it. It would be possible that this material was utilized as a thermistor and a polar solvent gas sensor in the relatively low temperature range.

\section{Experimental}

The stannous acetate powder of reagent grade was dried in a desiccator, ground fine in an agate mortar and then pressed into a tablet shape ( $1 \mathrm{~mm}$ in thickness and $10 \mathrm{~mm}$ in diameter) at $200 \mathrm{~kg} \cdot \mathrm{cm}^{-2}$ under vacuum. In order to measure the electric resistance, gold was evaporated in a vacuum bell jar as the electrodes which were separated with a gap of $5 \mathrm{~mm}$. Thin nickel wires were used as the leads contacted with the electrodes by use of a commercial silver paste. A tablet sample was put on a Pyrex holder in which a thermocouple was set (Fig. 1 (A)) and introduced into an electric furnace. Electric resistance was obtained by measuring the current at a constant voltage of $50 \mathrm{mV}$. The electric circuit is schematically shown in Fig. 1 (B).

\section{Results and Discussion}

\subsection{Effect of temperature on resistivity} of pressed stannous acetate powder

Change in the electric resistance of the tablet sample by repeated heating and cooling was recorded. The temperature was limited below $453 \mathrm{~K}$, the melting point of stannous acetate. Figure 2 shows a response of electric resistance of a sample to the cyclic temperature change. It is evidently seen that the change in electric resistance corresponds with that in temperature. In this temperature range, X-ray diffraction and IR spectrum did not detect any other substances except stannous acetate. It is, therefore, evident that stannous acetate possesses the semiconductive properties.

In general, the electric resistance of semiconductors, $R$ can be expressed as in the following equation,

$$
R=R_{0} \exp \left(B\left(1 / T-1 / T_{0}\right)\right),
$$

where $R_{0}$ is the resistance at $T_{0}(\mathrm{~K})$ and $B$ is 


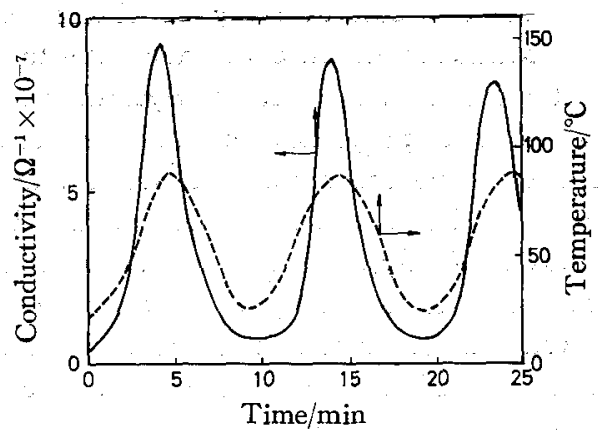

Fig. 2 Response of electric resistance of stannous acetate to cyclic change of temperature in low temperature range

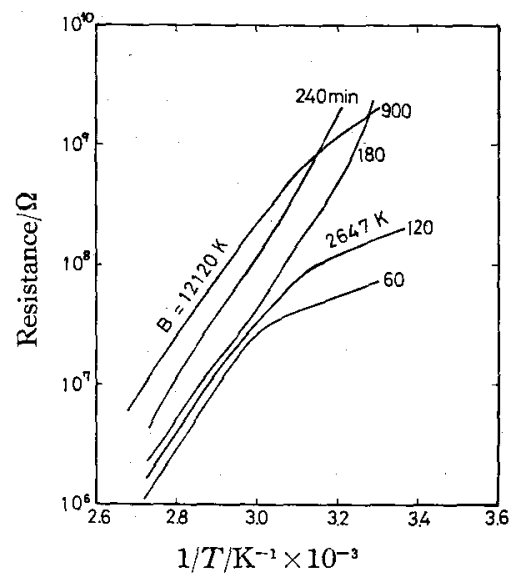

Fig. 3 Effect of preliminary powder-baking time on resistance-1/ $T$ relation of pressed stannous acetate tablets

referred to as "thermistor constant". The values of $R_{0}$ and $B$ obtained for the pressed tablet samples greatly changed with the advancement in heating cycles. It was found from the weight loss measurement with a thermobalance that the excess acetic acid evaporated from the reagent stannous acetate powder above $317 \mathrm{~K}$. To obtain the reproducible data, the stannous acetate reagent powder was baked in the flow of a refined nitrogen gas prior to the pressforming. Figure 3 shows the effect of baking time at $393 \mathrm{~K}$ on $R-1 / T$ plots according to eq. (1). The samples made from the powder baked for more than 3 hours indicated good linearity with a stable thermistor constant of about $12,000 \mathrm{~K}$, which was among the biggest values for the known organic semiconductive materials?).
Table 1 Solvents used for study on doping effect on electric conductivity of pressed stannous acetate powder and their specific dielectric constants $^{(0)}$

\begin{tabular}{|c|c|c|c|}
\hline \multicolumn{2}{|c|}{ Increasing } & \multicolumn{2}{|c|}{ No effect } \\
\hline Substance & $\begin{array}{l}\text { Specific } \\
\text { dielectric } \\
\text { constant }\end{array}$ & Substance & $\begin{array}{l}\text { Specific } \\
\text { dielectric } \\
\text { constant }\end{array}$ \\
\hline Acetone & $\begin{array}{l}17.39 \\
(289 \mathrm{k})\end{array}$ & Benzene & $\begin{array}{c}2.27 \\
(298 \mathrm{k})\end{array}$ \\
\hline Aniline & $\begin{array}{c}6.89 \\
(293 k)\end{array}$ & Cyclohexane & $\begin{array}{c}2.20 \\
(298 \mathrm{k})\end{array}$ \\
\hline $\begin{array}{l}\text { Acetic } \\
\text { I'janhydride }\end{array}$ & $\begin{array}{c}20.7 \\
(292 \mathrm{k})\end{array}$ & Cyclohexanone & $\begin{array}{c}18.3 \\
(293 \mathrm{k})\end{array}$ \\
\hline Glycerin & $\begin{array}{c}42.5 \\
(298 \mathrm{k})\end{array}$ & $\begin{array}{l}\text { Carbontetra- } \\
\text { chloride }\end{array}$ & - \\
\hline Methanol & $\begin{array}{l}32.63 \\
(298 \mathrm{k})\end{array}$ & Ethylether & $\begin{array}{c}4.20 \\
(300 \mathrm{k})\end{array}$ \\
\hline Water & $\begin{array}{c}78.54 \\
(298 \mathrm{k})\end{array}$ & Oleic acid & $\begin{array}{c}2.46 \\
(293 \mathrm{k})\end{array}$ \\
\hline & & Toluene & $\begin{array}{c}2.38 \\
(298 k)\end{array}$ \\
\hline
\end{tabular}

However, $R_{0}$ still increased with increasing baking time over 3 hours. This was probably because the excess acetic acid contained in the powder was not completely removed. Baking of stannous acetate powder for the extended time led to improvement in the reproducibility, while the value of $B$ and $R_{0}$. became one to two orders of magnitude larger than those for the samples without being baked. However, no difference was detected in $\mathrm{X}$-ray diffraction or $I R$ spectrum between the baked and unbaked powder tablets.

\subsection{Doping effect of polar solvents on electric resistance}

The specific resistance of the baked and then pressed stannous acetate powder tablets was as small as $400-800 \mathrm{~K} \Omega \cdot \mathrm{cm}$ at $293 \mathrm{~K}$. Getting a hint from the experimental fact that the excess acetic acid had great effect on the values of $B$ and $R_{0}$, a drop of some other solvents listed in Table 1 were attempted to be added on the baked and pressed tablet samples. It is found that the penetration of the polar solvents into the tablet samples resulted in a remarkable decrease of the electric resistance and that the extent of the decrease roughly depended on the specific dielectric constants of the polar 


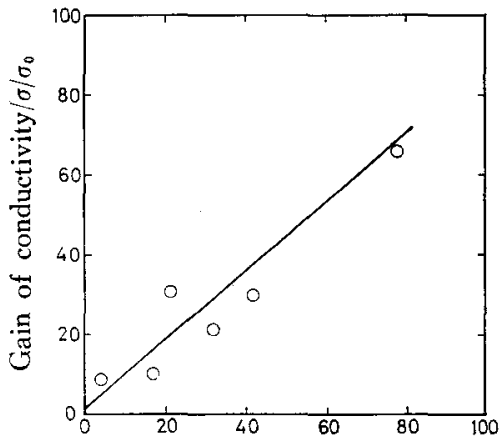

Specific dielectric constant $/ \varepsilon / \varepsilon_{0}$

Fig. 4 Effect of specific dielectric constant of doped solvents on increase in electric conductivity of stannous acetate at $298 \mathrm{~K}$

solvents tested (Fig. 4). Among the tested solvents, glycerin was the most stable and effective material for modification of the semiconductive properties because it had the lowest vapor pressure below $423 \mathrm{~K}(133 \mathrm{~Pa}$ at $400 \mathrm{~K})$ and decreased $R_{0}$ by about 30 times $\left(\mathrm{T}_{0}=293 \mathrm{~K}\right.$ ) compared with the samples without any solvents. However, it also decreased $B$ from 12,000 to $4,610 \mathrm{~K}$. The latter value was among the similar magnitude for some of commercial glass thermistors ${ }^{5}$.

\subsection{Measurement of thermoelectric motive force}

Measurement of Hall coefficient was attempted, but failed on account of too high resistance. However, thermoelectric motive force could be measured for the baked stannous acetate powder tablets with and without doping of glycerin. The Zeebeck coefficient, $(\mathrm{d} V / \mathrm{d} T)^{* 1}$ obtained for the tablet with glycerin was $-0.041 \mathrm{mV}$. $\mathrm{deg}^{-1}$ and for that without glycerin -0.087 $\mathrm{mV} \cdot \mathrm{deg}^{-1}$ around $288 \mathrm{~K}$. The negative value of the Zeebeck coefficient suggested an $n$-type semiconductor and the decrease in its absolute value by doping glycerin meant increase in electron density of the conduction band ${ }^{6}$. Therefore, it seems that glycerin acts as an electron donor molecule.

\subsection{The role of polar solvents on the}

\footnotetext{
*1 The ohmic contact between the gold electrodes and the stannous acetate tablet is satisfied because the voltage current relation obeyed the Ohm's law in the applied voltage range from -1.0 to $1.0 \mathrm{~V}$.
}

electric conductivity of the pressed stannous acetate powder tablets

As for the doping effect of glycerin on the semiconductive properties of stannous acetate, several cases are conceivable. It seems that the effect of polar solvents can be explained by two ways. Firstly, it is due to the ionic conduction of the doped polar solvents or the dissolved stannous ions and secondly, to adsorption or orientation of the polar solvent molecules on the mutually contacting powder interfaces.

In the first case, electrode reaction should take place on the gold electrodes. The most possible reaction seems to be the redox reaction between stannous and stannic ions. The temperature dependence of the specific resistance of pure water, for example, gives that $B$ value of $4,350 \mathrm{~K}$ according to a hand book $^{7}$, which is the same order of magnitude to those for the polar solvent-doped tablets. Therefore, it would be possible that ionic conduction interprete the doping effect of the polar solvents.

In the second case, an explanation of the doping effect by change in electronic conductivity is also possible because the glycerin-doped stannous acetate tablet had a thermoelectric motive force and the thermistor constant corresponded to those of commercial glass thermistors. Therefore, it is likely that the contact of stannous acetate powders plays an important role on controlling the electric conductivity. By this concept ${ }^{8)}$, a potential barrier is established in the interface of a powder contact due to existence of the surface levels which are induced by crystal imperfections or impurities. When electron donor molecules are adsorbed on the interface of the $n$-semiconductive powder contact and occupy the surface accepter levels, the potential barrier will be reduced. Figure 5 shows the change in the potential barrier built in the vicinity of the powder contacting interface $(n-n$ junction) by adsorption of the electron donor molecules.

The current flowing over the potential barrier may be written as follows if a voltage $V$ is applied over the tablet sample,

$$
\begin{aligned}
I= & A \cdot \exp (-\phi / k T) \cdot\{\exp (\mathrm{eV} / 2 N k T) \\
& -\exp (-\mathrm{eV} / 2 N k T)\}
\end{aligned}
$$

where $A$ is a constant so long as the powder contacting area and the electron density are 


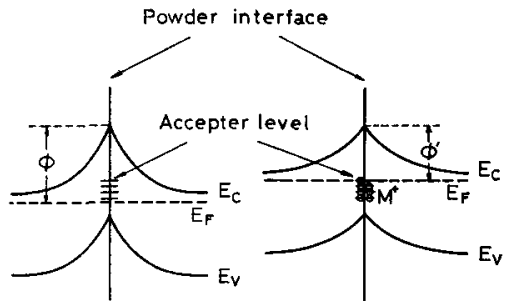

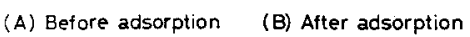

Fig. 5 Change in potential barrier established in contacting interface of powdery $\mathbf{n}$ type semiconductors by adsorption of electron-donor molecules

unchanged, $\phi$ is potential barrier, $N$ is the number of powder contacts in the currentflowing direction between the electrodes and the other symbols have their usual meanings. $V / 2 \mathrm{~N}$ means the voltage over one side of a potential barrier. Since $V / N$ is extremely small, eq. (2) will be reduced to

$$
I=(A \mathrm{eV} / N k T) \exp (-\phi / k T)
$$

Assuming that the whole resistance of the pressed stannous acetate powder sample is mainly attributed to the potential barrier at the interface of powder contacts. It may be expressed as a series connection of each interface resistance according to eq. (3),

$$
R_{t}=V / I=(N k T / \mathrm{e} A) \exp (\phi / k T)
$$

The whole resistance $R_{t}$ holds the Ohm's law and is nearly in proportion to $1 / T$ in the narrow potential range. The adsorption of the polar solvents which have ability to donate electrons on the interface reduces the potential barrier from $\phi$ to $\phi^{\prime}$ (Fig. 5) and augments $A$ due to increase in the electron density. These discussions can qualitatively explain the experimental results that the electric resistance and thermistor constant were decreased by doping of glycerin.

For satisfaction of the above discussion, the stannous acetate crystals should have much larger conductivity than their contacting interfaces.
Unfortunately, the semiconducting properties of stannous acetate bulk has not been measured. However, it would be possible that the stannous acetate crystals possessed the strong semiconductive properties due to something like "hopping mechanism", taking into consideration the report by Donaldson et $a l^{9}$ that stannous acetate was a fibrous molecule which contained $4 \%$ stannic ions against stannous ions.

At the present time, it is difficult to determine which mechanism of ionic conductivity or adsorption controls the doping effect of the polar solvents. Further detailed data should be required for this purpose.

\section{Conclusion}

The pressed stannous acetate powder was found to have semiconductive properties. Especially, it indicated the excellent sensitivity to the temperature change. The thermoelectric motive force measurement revealed that stannous acetate possessed the $n$-type semiconductivity. Doping of the polar solvents gave the strong effect on its conductivity. Especially, glycerin greatly strengthened the $n$-type semiconductivity.

\section{Reference :}

1) E. Kawamata, S. Kambe and K. Oshima, Oyo Butsuri 44, 742 (1975).

2) E. Kawamata, S. Kambe and K. Oshima, Oyo Butsuri 46, 490 (1977).

3) E. Kawamata, S. Kambe and K. Oshima, Oyo Butsuri 47, 16 (1978).

4) M. Hatano and S. Kanbara, Kobunshi 9, 972 (1960).

5) "Semiconductors Hand Book" Ohm, 1977, p. 464.

6) T. Kawaguchi, "Chemistry of Semiconductors", Maruzen, 1974, p. 50.

7) “Kagaku binran", Chem. Soc. Japan, 1975, p. 1181.

8) K. Takahashi, "Introduction of Sensoring Technique”, Kogyo Chosakai, 1979, p. 127.

9) J.D. Donaldson, W. Moser and W.B. Simpson, $J$. Chem. Soc., Suppl. 2, 5942 (1964).

10) "Kagaku binran", Chem. Soc. Japan, 1975, p. 1166.

(Received Aug. 26, 1981; Accepted Jan. 19, 1982) 